\title{
Guest Editorial: The Health of S Asian Communities in the U.S.
}

\author{
Anna Banerji ${ }^{1,2,3}$ \\ Published online: 19 July 2019 \\ (c) Springer Science+Business Media, LLC, part of Springer Nature 2019
}

With approximately 1 billion people on Earth who are of South Asian (SA) heritage, the SA diaspora has diffused to all corners of the globe, with greater than 2.5 million SAs living in the USA. This includes migrants and their descendants from India, Pakistan, Bangladesh, Sri Lanka and Nepal. While SAs are a diverse and complex group, with unique health risk factors, they are over-represented for conditions such as cardiovascular disease (CV), metabolic syndromes and cancer risk and mental health issues. Most of the literature addressing health and health concerns of SAs aggregates the population and does not reflect the unique diversities of religion, culture, gender, generation and/or country specific or region-specific characteristics that impact health risks. There is a paucity of literature which seeks though a cultural lens to assess the risk and protective factors for disease in South Asian immigrant (SAI) communities. The question remains, what are the different belief systems and cultural values that are prevalent in the diverse subgroups that impact health risk, and access to healthcare? Recently a collaborative effort of researchers, health practitioners and SA community partners resulted in the first translational research of its kind in the SA population.

South Asian Health: From Research to Practice and Policy created in 2013 (Gany), resulted from the partnership of the South Asian Health Initiative in New York and the Palo Alto Medical Foundation Research Institute in California which brought together research and evidence-based approaches to practice and policy for the SA migrant community with the intention of reducing the health disparities. Their objectives include the identification of risk factors for

Anna Banerji

anna.banerji@utoronto.ca

1 Indigenous and Refugee Health, University of Toronto, Toronto, Canada

2 Faculty of Medicine, University of Toronto, Toronto, Canada

3 Dalla Lana School of Public Health, University of Toronto, 500 University Avenue, 6th Floor, Toronto, ON M5G 1V7, Canada conditions that disproportionately impact the SA migrant community, and the investigation of the unique social, cultural, religious, generational, gender-based differences impact health and healthcare access, and to reduce the health disparity in culturally appropriate ways.

This partnership identified several high priority themes to be evaluated. Through literature searches, virtual and inperson meetings, and from town-hall meetings with consultation with the SA community and leaders, the outcome is a series of papers which enhance our knowledge of the behavioral and belief systems that modify risks for $\mathrm{CV}$ disease, cancer and mental health in different subpopulations of SAI; provide culturally tailored prevention, diagnosis and treatment models; and inform future research. While the risk for disease is multifactorial and involves the interaction of genetics, environmental and lifestyle factors, this series highlights how the cultural, belief and value systems may impact behavioral and lifestyle risks for disease.

In general, SA cultures emphasize collectivism, where family objectives of the family are more important than individual objectives, which include family harmony, obedience to elderly. While this can be protective in dealing with stress, it can lead to unexpressed stress and conflict and inter-generational conflict. Fatalism, the idea that changing health outcomes is out of someone's control and that it is not possible to modify illness by behavior change, was a prevalent theme in many SA communities and is a barrier to preventing lifestyle change. The degree of fatalism appeared to decrease with the degree of acculturation. While religiosity may be a protective coping mechanism, it may also be associated with fatalism. The belief that one cannot prevent $\mathrm{CV}$, diabetes, or cancer can be a barrier to modifying behavior, and needs to be understood to develop culturally appropriate educational material.

SA migrants are a complex group with multiple subcategories with varying risks for exposures. Some risks are generalizable to the majority of the population; for example, there is a great burden of disease from Hepatitis $\mathrm{B}$ and cervical cancer and CV disease. Other risks, however, may be more likely to affect specific subgroups 
because of certain characteristics, e.g., arsenic toxicities in Bangladeshis, increased risks attributable to religion, degree of acculturation, gender, age, and/or occupation (such as taxi drivers). The complexity can be illustrated by alcohol, which is a risk for CV disease, obesity and cancer. Alcohol is permitted in Hinduism, but not in the Muslim or Jain religions. Although first generation SA women tend not to drink, alcohol may be a risk factor for breast cancer in successive generations of SA women.

Kandula et al. investigated the impact of sociocultural influences on lifestyle and behavior that impact the risk of CV disease and cancer risk among South Asians. SAI immigrants have elevated rates of CV disease as well as type 2 diabetes, obesity and metabolic syndromes. Inadequate physical activity is a known significant independent risk factor for CVD, obesity, and other metabolic syndromes. Kandula et al. describe values and beliefs that could impede the adaption of physical activity. Pressures in the SA community to fulfill social and financial obligations lead to a view of physical activity as selfish and a low priority and it is often not supported family members. Women participate less due to religious modesty, avoidance of mixed-sex activities, and cultural stigma, whereas youth are often encouraged to spend time on studying or going to school to meet family obligations. Vigorous physical activity was often associated with "paid labour" and thus not desirable. Fatalism led to the belief that the risk for cardiovascular disease was not modifiable through exercise or dietary changes and is a barrier to risk reduction.

Karasz et al. describe the conceptualization of mental health and stress among SA. SAI like many immigrant communities experience migration stress, unemployment, poverty, acculturation stress, language barriers, and discrimination. There are high rates of mental health issues in SAI which often remain undiagnosed, with variations across ethnicities, gender and generational subgroups. Depression is the most common mental health diagnosis, with increased risks due to older age, low literacy, financial difficulties, social isolation and poor physical health. In contrast to North America, the suicide rate of SAI women is much greater than that of SAI men. SA women often experience cultural conflicts in gender roles. The emphasis on collectivism rather than individualism can lead to the suppression of women and younger family members and lead to unexpressed stress and conflict. Predictors of mental distress among SA women include a history of domestic violence, marital conflict, financial coercion, and isolation. Among SA youth, mental stress is associated with poor acculturation, discrimination, high parental expectations, poor self-esteem, domestic violence. Risk factors for mental stress among older adults include abuse, neglect, social isolation, acculturation stress, and, poor physical health. Traditional homes are more likely to experience intergenerational conflict and have a higher prevalence of eating disorders.
Mental illness is often seen as the failure of a family to solve a problem, and religious beliefs and fatalism deter people from seeking professional health. Social stigma and shame were identified as a major barrier for not seeing a mental health professional. SAs with mental illness commonly interpret their symptoms as physical illness (which is more acceptable) and do not seek psychological help. SAs caring for older adults with dementia often did not consult a professional. Additionally, members of the SAI community may hold the belief that mental health or suicide prevention strategies are not beneficial. Lack of cultural responsiveness among providers, assumptions related to normative gender or family roles, and religious or cultural beliefs may be a barrier to accessing diagnosis and treatment. Relevant cultural training for health providers may improve diagnosis and treatment.

Leung et al. discuss infections and chronic inflammation that can increase the risk for $\mathrm{CV}$ disease and cancer in SAI. This paper also highlights the diversity of risks from diet and related exposures, including fasting, vegetarianism, use of chewing tobacco, alcohol, and alternative medicine, all of which vary depending on ethnicity, religion, sex, degrees of religiosity and fatalism with generational and levels of acculturation. Such variations are observable in large populations. As examples, India has one-quarter of the global burden of disease for cervical cancer, with the highest mortality rates in the world, while Bangladeshis are at elevated risk of arsenic toxicity and cancers and CAD from drinking water or eating crops derived from contaminated ground. Indian and Pakistani immigrants have cancer profiles reflective of the country of origin with oral and cervical cancers having the highest incidence in men and women respectively. However, immigrants from these countries to the United States begin to reflect the patterns of cancer within the larger U.S. population over a period of time and with increased levels of acculturation to the lifestyle practices of the local population. The risk may be compounded by religion and gender; for example, Hindu men may drink alcohol, and exposure to oral tobacco (paan) and cigarettes exponentially increases the risk of oral cancer.

These series of papers provide important insights into some of the diverse risks, perspectives and beliefs impacting lifestyle, facilitating the identification of research gaps, the formulation of relevant policy, and the promotion of education that can ameliorate risks, enhance protective factors, and health to reduce the health disparities experienced by SA immigrants and communities.

Publisher's Note Springer Nature remains neutral with regard to jurisdictional claims in published maps and institutional affiliations. 